\title{
The Entropy Density of Quasi Free States
}

\author{
M. Fannes ${ }^{\star}$ \\ Universiteit Leuven, Belgium \\ Received September 5, 1972
}

\begin{abstract}
For lattice invariant quasi free states on the Fermi lattice system the mean entropy is explicitly calculated; it is proved that it is a norm continuous functional on this set of states which is not weakly continuous.
\end{abstract}

\section{Introduction}

In the algebraic approach of statistical mechanics a lot of work has been done on the existence and properties of the mean entropy of translation invariant states (Ref. [1,2]). For classical systems and quantum lattice systems the existence of mean entropy has been proved; furthermore as fundamental properties it has been proved that the entropy-functional on the set of invariant states is affine and semicontinuous.

In this work we study the mean entropy of the quasi-free lattice invariant states on the Fermi lattice system. The existence of the mean entropy follows from Ref. [2]. Here we derive an explicit form of the mean entropy for those states (Theorem 2). This result may be usefull in deriving rigorous results for solvable models in statistical mechanics.

Furthermore much attention is given to the continuity properties of the entropy functional with respect to different topologies on the set of quasi-free states. It is proved that it is continuous with respect to the norm topology (Theorem 1) and that it is not continuous with respect to the weak topology (Theorem 3).

\section{Entropy density}

\section{A. Preliminaries}

1. The Fermi Lattice Algebra

We consider the one-dimensional lattice $\mathbb{Z}=\{0, \pm 1, \pm 2, \ldots\}$. The Hilbert space $l^{2}(\mathbb{Z})$ can also be considered as an Euclidian space $H$

* Navorsingsstagiair van het Belgisch N.F.W.O. 
equipped with the inner product:

$$
s(\psi, \phi)=\operatorname{Re}\langle\psi, \phi\rangle
$$

where $\langle\ldots\rangle$ is the ordinary complex inner product.

Let $\Lambda \subset \mathbb{Z}$ be a finite set, then

$$
l^{2}(\Lambda) \subset l^{2}(\mathbb{Z})
$$

and the corresponding Euclidean space $H_{A} \subset H$.

It is clear that

$$
H=\bigcup_{A \subset \mathbb{Z}} H_{A} \text { where - means the closure. }
$$

To every finite $\Lambda \subset \mathbb{Z}$ we associate the C.A.R. algebra $\mathscr{A}_{A}$ of observables of the volume $A$. It is generated as a complex algebra by the elements $B(\phi) ; \phi \in H_{\Lambda}$. The $r$-linear map $\phi \rightarrow B(\phi)$ satisfies:

i) $B^{*}(\phi)=B(\phi)$,

ii) $\{B(\phi), B(\psi)\}=2 s(\phi, \psi)$.

If $A_{1} \subset A_{2}$ then there exists an isometric injection $\mathscr{A}_{\Lambda_{1}} \rightarrow \mathscr{A}_{A_{2}}$.

Hence $\mathscr{A}=\bigcup_{A \subset \mathbb{Z}} \mathscr{A}_{\Lambda}$ can be equipped with a $C^{*}$-norm compatible with the norms on each $\mathscr{A}_{A}$.

$\overline{\mathscr{A}}=\bigcup_{A \subset \mathbb{Z}} \mathscr{A}_{A}^{n}$ is called the Fermi lattice algebra of the quasi-local observables. Furthermore, if $\overline{\mathscr{A}(H, s)}$ is the C.A.R. algebra [3] constructed on $H$, we have $\overline{\mathscr{A}}=\overline{\mathscr{A}(H, s)}$ because they contain both the same dense algebra generated by the set $\left\{B(\phi), \phi \in \bigcup_{\Lambda \subset \mathbb{Z}} H_{\Lambda}\right\}$.

\section{Lattice Invariant Quasi Free States}

Let $e^{i} \in l^{2}(\mathbb{Z})$ be the sequence $\left(\delta_{i, x}\right)_{x \in \mathbb{Z}}$ and $f^{2 n-1}=e^{n} ; f^{2 n}=i e^{n}, n \in \mathbb{Z}$. The set $\left\{e^{i}, i \in \mathbb{Z}\right\}$ is a complete orthogonal basis of $l^{2}(\mathbb{Z})$ and so is $\left\{f^{n}, n \in \mathbb{Z}\right\}$ for $H$.

We define a s-orthogonal operator $T \in B(H)$, called the translation operator, by:

$$
T f^{n}=f^{n+1}, \quad n \in \mathbb{Z} .
$$

$T$ induces an automorphism $\tau_{T}$ of $\overline{\mathscr{A}}$ defined by [4]:

$$
\tau_{T} B(\phi)=B(T \phi), \quad \phi \in H .
$$

A state $\omega$ on $\overline{\mathscr{A}}$ is called translation invariant if

$$
\omega\left(\tau_{T} x\right)=\omega(x), \quad x \in \overline{\mathscr{A}} .
$$


In what follows we restrict ourselves to the set of quasi free states $\omega_{A}$ on $\overline{\mathscr{A}}$. They are uniquely determined by their two-point functions:

$$
\omega_{A}(B(\phi) B(\psi))=s(\phi, \psi)+i s(A \phi, \psi), \quad \phi, \psi \in H,
$$

where $A \in B(H)$ (all bounded operators on $H$ ) satisfies

$$
\begin{array}{cl}
A^{+}=-A & \left(A^{+}=\text {adjoint of } A \text { with respect to } s\right), \\
& 0 \leqq A^{+} A \leqq 1 .
\end{array}
$$

The quasi free state $\omega_{A}$ is translation invariant iff $[A, T]=0$, i.e. iff the operator $A$ is translation invariant [4].

Translation invariant operators can be described as multiplication operators as follows [5]: let $\mathbb{C}_{1}$ be the circle of radius $1 / 2 \pi$ and $L^{2}\left(\mathbb{C}_{1}\right)$ the real Hilbert space of square integrable functions on $\mathbb{C}_{1}$. The map $U$ of $H$ into the underlying real subspace of $L^{2}\left(\mathbb{C}_{1}\right)$ defined by

$$
U f^{n}=\hat{f}^{n}(x)=e^{2 \pi \imath n x} \quad \text { is unitary . }
$$

If $[A, T]=0$ and $f \in L^{2}\left(\mathbb{C}_{1}\right)$ then:

i) $(\hat{A} f)(x)=\left(U A U^{*}\right) f(x)=a(x) f(x)$ where $a(x)=\sum_{k \in \mathbb{Z}} s\left(f^{k}, A f^{0}\right) \hat{f}^{k}(x)$,

ii) $\left(\hat{A}^{+} f\right)(x)=\overline{a(x)} f(x)=a(1-x) f(x)$,

iii) $\|A\|=\sup _{x \in \mathbb{C}_{1}}|a(x)|$.

Hence we see that each quasi free state $\omega_{A}$ which is translation invariant defines a multiplication operator $\hat{A}$ :

with

$$
(\hat{A} f)(x)=a(x) f(x)=i b(x) f(x)
$$

$$
\begin{aligned}
& x \rightarrow b(x) \quad \text { real, } \\
b(x)= & -b(1-x), \\
|b(x)| \leqq 1 . &
\end{aligned}
$$

\section{Definition of the Local Entropy}

If $\Lambda \subset \mathbb{Z}$ is a finite set we denote by $N(\Lambda)$ the number of points in $\Lambda$. For a translation invariant state $\omega$ the existence of the entropy density

$$
s(\omega)=\lim _{\Lambda \rightarrow \infty} s_{\Lambda}(\omega)=\lim _{\Lambda \rightarrow \infty} \frac{S_{\Lambda}(\omega)}{N(\Lambda)}
$$

has been proved [2], where $S_{A}(\omega)$ is the local entropy which can be written in the following form if $\omega=\omega_{A}$ is a quasi free state [6]:

$$
S_{\Lambda}\left(\omega_{A}\right)=-\frac{1}{2} \operatorname{Tr}_{H_{A}}\left\{\left(\frac{1+\left|A_{A}\right|}{2}\right) \log \left(\frac{1+\left|A_{\Lambda}\right|}{2}\right)+\left(\frac{1-\left|A_{\Lambda}\right|}{2}\right) \log \left(\frac{1-\left|A_{\Lambda}\right|}{2}\right)\right\}
$$


where

$$
\begin{aligned}
\left|A_{\Lambda}\right| & =\left(A_{\Lambda}^{+} A_{\Lambda}\right)^{1 / 2} \\
A_{\Lambda} & =P_{\Lambda} A P_{A}
\end{aligned}
$$

and $P_{A}$ is the projection operator on $H_{A}$.

In the following we derive an explicit expression for the entropy density $s\left(\omega_{A}\right)$ of a translation invariant quasi free state $\omega_{A}$ in terms of the associated multiplication operator $\hat{A}$. We denote by $\mathscr{E}$ the set of translation invariant quasi free states on $\overline{\mathscr{A}}$.

\section{B. A Continuity Property of the Entropy Density}

Define a continuous real function $f:[0,1] \rightarrow[0, \log 2]$ by:

$$
\begin{aligned}
& f(x)=-\left(\frac{1+x}{2}\right) \log \left(\frac{1+x}{2}\right)-\left(\frac{1-x}{2}\right) \log \left(\frac{1-x}{2}\right), \quad 0 \leqq x<1, \\
& f(1)=0 .
\end{aligned}
$$

Lemma 1. $|f(x)-f(y)| \leqq f(1-|x-y|), x, y \in[0,1]$.

Proof. The case $x=y$ is trivial. Suppose $y>x$ and put $\varepsilon=y-x>0$. The case $\varepsilon=1[y=1, x=0]$ is again trivial. Hence we suppose $0<\varepsilon<1$.

Now:

$$
\frac{d f(x)}{d x}=\frac{1}{2} \log \frac{1-x}{1+x}<0 \text { for } x \in(0,1) .
$$

hence $x \rightarrow f(x)$ is a monotonically decreasing function of $x \in[0,1]$ and:

$$
|f(x)-f(y)|=f(x)-f(y)=f(x)-f(x+\varepsilon)=F_{\varepsilon}(x) .
$$

The function $x \rightarrow F_{\varepsilon}(x)$ is defined and continuous for $x \in[0,1-\varepsilon]$ and:

$$
\frac{d F_{\varepsilon}(x)}{d x}=\frac{1}{2} \log \frac{(1-x)(1+x+\varepsilon)}{(1+x)(1-x-\varepsilon)}>0 \text { for } x \in(0,1-\varepsilon) .
$$

Hence $x \rightarrow F_{\varepsilon}(x)$ is monotonically increasing for $x \in[0,1-\varepsilon]$ and thus:

$$
F_{\varepsilon}(x) \leqq F_{\varepsilon}(1-\varepsilon)=f(1-\varepsilon)
$$

The lemma follows from (1) and (2).

Theorem 1. The entropy functional $s: \mathscr{E} \rightarrow[0, \log 2]$ is continuous with respect to the norm topology on $\mathscr{E}$.

Proof. The map $\omega_{A} \in \mathscr{E} \rightarrow A \in B(H)$ is continuous with respect to the norm on $\mathscr{E}$ and $B(H)$. We prove now that the map $A \rightarrow s\left(\omega_{A}\right)$ is norm continuous. $A \subset \mathbb{Z}$ is a finite volume.

If $S \in B\left(H_{\Lambda}\right)$ is a symmetric operator we denote by $\mu_{n}[S], 1 \leqq n \leqq 2 N(\Lambda)$ the eigenvalues of $S$ in ascending order. 
If $S, T \in B\left(H_{A}\right)$ are symmetric then ([7], Theorem 6.44):

$$
\left|\mu_{n}[S]-\mu_{n}[T]\right| \leqq\|S-T\|, \quad 1 \leqq n \leqq 2 N(\Lambda) .
$$

Let $\omega_{A}$ and $\omega_{B}$ be elements of $\mathscr{E}$ such that $\left\|\omega_{A}-\omega_{B}\right\| \leqq \frac{\varepsilon}{2}, \varepsilon \leqq 1$, then $\|A-B\| \leqq \frac{\varepsilon}{2}$.

Using Lemma 1 we get:

$$
\begin{aligned}
\left|S_{\Lambda}\left(\omega_{A}\right)-S_{\Lambda}\left(\omega_{B}\right)\right| & \leqq \frac{1}{2}\left|\sum_{n=1}^{2 N(\Lambda)} f\left(\mu_{n}\left[\left|A_{\Lambda}\right|\right]\right)-f\left(\mu_{n}\left[\left|B_{\Lambda}\right|\right]\right)\right| \\
& \leqq \frac{1}{2} \sum_{n=1}^{2 N(\Lambda)}\left|f\left(\mu_{n}\left[\left|A_{\Lambda}\right|\right]\right)-f\left(\mu_{n}\left[\left|B_{\Lambda}\right|\right]\right)\right| \\
& \leqq \frac{1}{2} \sum_{n=1}^{2 N(\Lambda)} f\left(1-\left|\mu_{n}\left[\left|A_{\Lambda}\right|\right]-\mu_{n}\left[\left|B_{\Lambda}\right|\right]\right|\right) .
\end{aligned}
$$

Now for $x, y \geqq 0$ we have $|x-y| \leqq \sqrt{\left|x^{2}-y^{2}\right|}$, hence:

$$
\begin{aligned}
\left|S_{\Lambda}\left(\omega_{A}\right)-S_{\Lambda}\left(\omega_{B}\right)\right| & \leqq \frac{1}{2} \sum_{n=1}^{2 N(A)} f\left(1-\sqrt{\mid \mu_{n}\left[\left|A_{A}\right|^{2}\right]-\mu_{n}\left[\left|B_{A}\right|^{2}\right]}\right) \\
& \leqq N(\Lambda) f\left(1-\sqrt{\|\left|A_{\Lambda}\right|^{2}-\left|B_{A}\right|^{2} \mid}\right) .
\end{aligned}
$$

Furthermore we have:

and hence:

$$
\begin{aligned}
\left\|\left|A_{\Lambda}\right|^{2}-\left|B_{\Lambda}\right|^{2}\right\| & =\left\|A_{\Lambda}^{+}\left(A_{\Lambda}-B_{\Lambda}\right)+\left(A_{\Lambda}^{+}-B_{\Lambda}^{+}\right) B_{\Lambda}\right\| \\
& \leqq\left(\left\|A_{\Lambda}\right\|+\left\|B_{\Lambda}\right\|\right)\left\|A_{\Lambda}-B_{\Lambda}\right\| \\
& \leqq 2\|A-B\| \leqq \varepsilon \leqq 1
\end{aligned}
$$

$$
\left|s_{\Lambda}\left(\omega_{A}\right)-s_{\Lambda}\left(\omega_{B}\right)\right| \leqq f(1-\sqrt{2\|A-B\|}) \text {. }
$$

\section{Explicit Form of the Entropy Density}

Lemma 2. Let

$$
f_{k}\left(x_{1}, \ldots, x_{k} ; n\right)=\sum_{n_{1}+\cdots+n_{k}=1}^{n} \sum_{n_{1}+\cdots+n_{k-1}=1}^{n} \ldots \sum_{n_{1}=1}^{n}\left(\prod_{l=1}^{n} e^{-i 2 \pi n_{l} x_{l}}\right)
$$

then

$$
\begin{gathered}
f_{k+1}\left(x_{1}, \ldots, x_{k+1} ; n\right) \\
=e^{-i(n+1) \pi x_{k+1}} \frac{\sin \left(n \pi x_{k+1}\right)}{\sin \left(\pi x_{k+1}\right)} f_{k}\left(x_{1}-x_{k+1}, \ldots, x_{k}-x_{k+1} ; n\right) .
\end{gathered}
$$


Proof.

$$
\begin{aligned}
f_{k+1}\left(x_{1}, \ldots, x_{k+1} ; n\right) & \\
= & \sum_{n_{k+1}=1-n_{1}-\cdots-n_{k}}^{n-n_{1} \cdots n_{k}} e^{-i 2 \pi n_{k+1} x_{k+1}} f_{k}\left(x_{1}, \ldots, x_{k} ; n\right) \\
= & e^{-l(n+1) \pi x_{k+1}} \frac{\sin \left(n \pi x_{k+1}\right)}{\sin \left(\pi x_{k+1}\right)}\left(\prod_{l=1}^{k} e^{i 2 \pi x_{k+1} n_{l}}\right) f_{k}\left(x_{1}, \ldots, x_{k} ; n\right) \\
= & e^{-i(n+1) \pi x_{k+1}} \frac{\sin \left(n \pi x_{k+1}\right)}{\sin \left(\pi x_{k+1}\right)} f_{k}\left(x_{1}-x_{k+1}, \ldots, x_{k}-x_{k+1} ; n\right)
\end{aligned}
$$

and

$$
f_{1}\left(x_{1} ; n\right)=e^{-\imath(n+1) \pi x_{1}} \frac{\sin \left(n \pi x_{1}\right)}{\sin \left(\pi x_{1}\right)} .
$$

Lemma 3. Let $\boldsymbol{x} \in \mathbb{R}^{k}$ and

$$
\delta_{k}(\boldsymbol{x} ; n)=\left(\prod_{i=1}^{k} \frac{\sin n x_{\imath}}{\sin x_{\imath}}\right)\left(\frac{\sin \left(n \sum_{i=1}^{k} x_{i}\right)}{n \sin \left(\sum_{l=1}^{k} x_{\imath}\right)}\right) .
$$

Let $I_{\varepsilon}=\left\{\boldsymbol{x} \in \mathbb{R}^{k} \mid-\pi+\varepsilon \leqq x_{i} \leqq \pi-\varepsilon ; \quad i=1, \ldots, k ; 0<\varepsilon<\pi\right\}$ and let $f: \mathbb{R}^{k} \rightarrow \mathbb{R}$ be a bounded integrable function on $I_{\varepsilon}$, continuous at the origin, then:

$$
\lim _{n \rightarrow \infty} \int_{I_{\varepsilon}} d \boldsymbol{x} f(\boldsymbol{x}) \delta_{k}(\boldsymbol{x} ; n)=\pi^{k} f(0)
$$

Proof. Choose a $\delta$ satisfying $0<\delta<\pi-\varepsilon$ and such that $f$ is continuous in $I_{1}=I_{(\pi-\delta)}$. Put $I_{2}=I_{\varepsilon} / I_{1}$ (see Fig. 1 for the 2-dim. case).

We prove first that only the origin contributes to the integral.

The zero's of $\sin \left(\sum_{i=1}^{k} x_{i}\right)$ are located on the parallel planes $\sum_{i=1}^{k} x_{i}=n \pi$ $n \in \mathbb{Z}$. There are at most three of those planes $(n=-1,0,1)$ which intersect $I_{2}$. We divide $I_{2}$ in two regions $R_{1}$ and $R_{2}$ :

$R_{1}$ is such that it consists of strips containing the zero's of $\sin \left(\sum_{l=1}^{k} x_{i}\right)$

that $\int_{R_{1}} d x<\eta$ with $\eta>0$, arbitrarely small and

$$
\text { that }\left|\prod_{l=1}^{k} \sin x_{i} \cdot \sin \left(\sum_{l=1}^{k} x_{l}\right)\right|>\xi>0 \quad \text { in } \quad R_{2}=I_{2} / R_{1}
$$



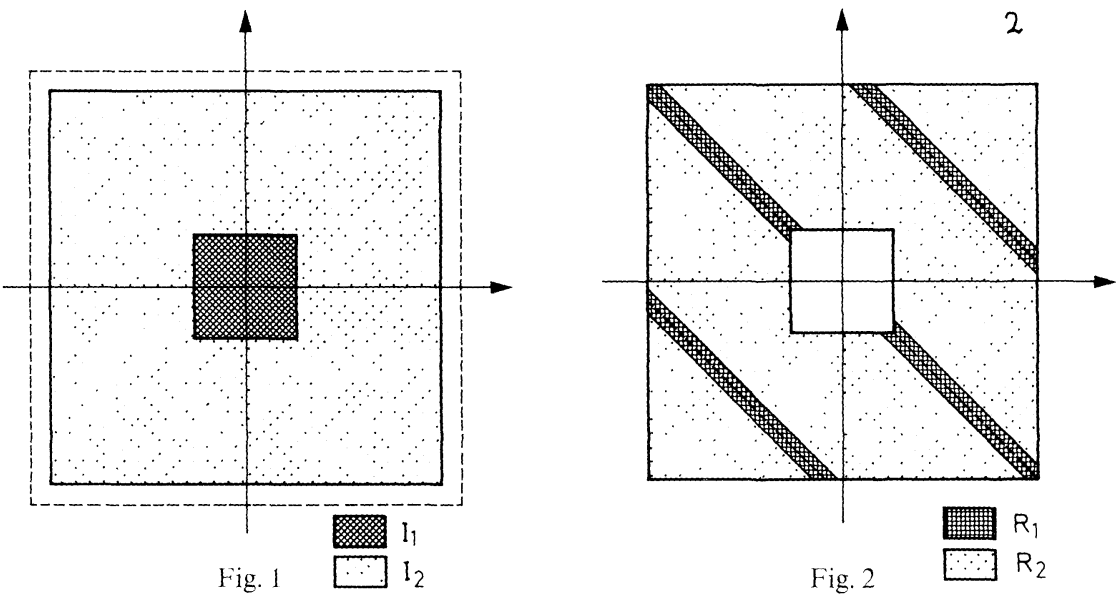

(see Fig. 2 for the 2-dim. case).

Then $\left|\frac{\sin \left(n \sum_{i=1}^{k} x_{i}\right)}{n \sin \left(\sum_{i=1}^{k} x_{i}\right)}\right| \leqq 1$ in $R_{1}$. So that

$$
\begin{aligned}
\delta_{k}(x ; n) & \text { is bounded by } \phi \text { in } R_{1} \text { and } \\
n \delta_{k}(x ; n) & \text { is bounded by } \frac{1}{\xi} \text { in } R_{2}
\end{aligned}
$$

both bounds are independent of $n$ and moreover $\phi$ is independent of $\eta$.

$$
\begin{aligned}
\left|\int_{I_{2}} d \boldsymbol{x} f(\boldsymbol{x}) \delta_{k}(\boldsymbol{x} ; n)\right| & \leqq\left|\int_{R_{1}} d \boldsymbol{x} f(\boldsymbol{x}) \delta_{k}(\boldsymbol{x} ; n)\right|+\frac{1}{n}\left|\int_{R_{2}} d \boldsymbol{x} f(\boldsymbol{x}) n \delta_{k}(\boldsymbol{x} ; n)\right| \\
& \leqq C\left(\eta \phi+\frac{V_{2}}{n \xi}\right)
\end{aligned}
$$

where $|f(x)| \leqq C$ for $x \in I_{\varepsilon}$ and $V_{2}=\int_{R_{2}} d x$.

Hence we have:

$$
\lim _{n \rightarrow \infty} \int_{I_{2}} d \boldsymbol{x} f(\boldsymbol{x}) \delta_{k}(\boldsymbol{x} ; n)=0 .
$$

Take now $\delta$ sufficiently small. Then $|f(x)-f(0)|<\alpha$ for $x \in I_{1}$ with $\alpha>0$ arbitrarely small.

$$
\left|\int_{I_{1}} d \boldsymbol{x} f(\boldsymbol{x}) \delta_{k}(\boldsymbol{x} ; n)-f(0) \int_{I_{1}} d \boldsymbol{x} \delta_{k}(\boldsymbol{x} ; n)\right| \leqq \alpha\left|\int_{I_{1}} d \boldsymbol{x} \delta_{k}(\boldsymbol{x} ; n)\right| .
$$


We estimate now

$$
A_{k}=\lim _{n \rightarrow \infty} \int_{I_{1}} d \boldsymbol{x} \delta_{k}(\boldsymbol{x} ; n) .
$$

Take a $\zeta>0$. Then for $\delta$ sufficiently small we have:

$$
\left|\int_{I_{1}} d \boldsymbol{x} \delta_{k}(\boldsymbol{x} ; n)-\int_{I_{1}} d \boldsymbol{x} \prod_{i=1}^{k} \frac{\sin \left(n x_{\imath}\right)}{x_{i}} \cdot \frac{\sin n\left(\sum x_{i}\right)}{n\left(\sum x_{i}\right)}\right|<\zeta
$$

because $x \rightarrow \frac{1}{\sin x}-\frac{1}{x}$ is continuous at $x=0$.

After the transformation $x_{i} \rightarrow n x_{i}$ :

$$
\int_{I_{1}} d x \prod_{i=1}^{k} \frac{\sin \left(n x_{i}\right)}{x_{i}} \cdot \frac{\sin n\left(\sum x_{i}\right)}{n\left(\sum x_{i}\right)}=\int_{-n \delta}^{+n \delta} d x \prod_{i=1}^{k} \frac{\sin x_{i}}{x_{i}} \cdot \frac{\sin \left(\sum x_{i}\right)}{\left(\sum x_{i}\right)}
$$

Then

Hence

$$
A_{k}=\int_{-\infty}^{+\infty} d x \prod_{i=1}^{k} \frac{\sin x_{i}}{x_{i}} \cdot \frac{\sin \sum x_{i}}{\sum x_{\imath}}=\pi^{k} .
$$

$$
\lim _{n \rightarrow \infty} \int_{I_{1}} d \boldsymbol{x} f(\boldsymbol{x}) \delta_{k}(\boldsymbol{x} ; n)=\pi^{k} f(0) .
$$

Combining (3) and (4) we get the desired result.

Now we calculate the entropy density in the case the multiplication operator $\hat{A}$ is a step function.

Lemma 4. Let $\omega_{A} \in \mathscr{E}$ be such that $\hat{A}$ is the multiplication operator by the step function $i b(x)$.

Let

$$
s^{\prime}(b)=-\int_{0}^{1} d x\left\{\left(\frac{1+b}{2}\right) \log \left(\frac{1+b}{2}\right)+\left(\frac{1-b}{2}\right) \log \left(\frac{1-b}{2}\right)\right\} .
$$

Then $s\left(\omega_{A}\right)=s^{\prime}(b)$.

Proof. Let $\Lambda_{n}=\{1, \ldots, n\} \subset \mathbb{Z} \quad n=1,2, \ldots$

For notational convenience note:

$$
\begin{aligned}
& P_{n}=P_{A_{n}} \quad \text { the projection on } H_{n}=H_{A_{n}} \\
& A_{n}=A_{\Lambda_{n}}=P_{n} A P_{n} ; S_{n}\left(\omega_{A}\right)=S_{\Lambda_{n}}\left(\omega_{A}\right) \text { and } S_{n}\left(\omega_{A}\right)=S_{\Lambda_{n}}\left(\omega_{A}\right) .
\end{aligned}
$$

Then

$$
\begin{aligned}
S_{n}\left(\omega_{A}\right) & =-\frac{1}{2} \operatorname{Tr}_{H_{n}}\left(\frac{1+\left|A_{n}\right|}{2}\right) \log \left(\frac{1+\left|A_{n}\right|}{2}\right)+\left(\frac{1-\left|A_{n}\right|}{2}\right) \log \left(\frac{1-\left|A_{n}\right|}{2}\right) \\
& =n \log 2-\frac{1}{2} \operatorname{Tr}_{H_{n}} \sum_{k=1}^{\infty} \frac{\left|A_{n}\right|^{2 k}}{2 k(2 k-1)} \\
& =n \log 2-\sum_{k=1}^{\infty} \frac{1}{2 k(2 k-1)} \frac{1}{2} \operatorname{Tr}_{H_{n}}\left|A_{n}\right|^{2 k} .
\end{aligned}
$$


The exchange of $\operatorname{Tr}$ with $\Sigma$ is allowed because $\operatorname{Tr}\left|A_{n}\right|^{2 k} \leqq 2 n$ and $\sum_{k=1}^{\infty} \frac{1}{2 k(2 k-1)}$ is convergent.

Note $\alpha_{n}(k)=\frac{1}{2 n} \operatorname{Tr}_{H_{n}}\left|A_{n}\right|^{2 k}$.

Then

$$
s_{n}\left(\omega_{A}\right)=\log 2-\sum_{k=1}^{\infty} \frac{1}{2 k(2 k-1)} \alpha_{n}(k) .
$$

First we look for $\lim _{n \rightarrow \infty} \alpha_{n}(k)$ for $k$ fixed.

$$
\begin{aligned}
\alpha_{n}(k) & =\frac{1}{2 n} \sum_{j=1}^{2 n} s\left(f^{j},\left(P_{n} A^{+} P_{n} A\right)^{k} P_{n} f^{j}\right) \\
& =\frac{1}{2 n} \sum_{j=1}^{2 n}\left(\hat{f}^{j},\left(\hat{B} \hat{P}_{n}\right)^{2 k-1} \hat{B} \hat{f}^{j}\right)
\end{aligned}
$$

where $\hat{B}$ is the multiplication by $b(x)$.

As $b(x)=\sum_{n \in \mathbb{Z}} b_{n} e^{i 2 \pi n x}$ we get

$$
\left(\hat{B} \hat{P}_{n}\right)^{2 k-1} \hat{B} \hat{f}^{j}=\sum_{n_{1}+\cdots+n_{2 k}+j=1}^{2 n} \ldots \sum_{n_{1}+j=1}^{2 n} b_{n_{1}} b_{n_{2}} \ldots b_{n_{2 k}} \hat{f}^{n_{1}+\cdots+n_{2 k}+j}
$$

and hence

$$
\begin{aligned}
\alpha_{n}(k) & =\frac{1}{2 n} \sum_{n_{1}+\ldots+n_{2 k}+j=1}^{2 n} \cdots \sum_{n_{1}+j=1}^{2 n} \sum_{j=1}^{2 n}\left(\prod_{l=1}^{2 n} \int_{0}^{1} d x_{l} b\left(x_{l}\right) e^{-i 2 \pi n_{l} x_{l}}\right) \delta_{n_{1}+\cdots+n_{2 k}, 0} \\
& =\frac{1}{2 n} \int_{0}^{1} \ldots \int_{0}^{1} d x_{1} \ldots d x_{2 k} b\left(x_{1}\right) \ldots b\left(x_{2 k}\right) g_{2 k}\left(x_{1}, \ldots, x_{2 k} ; 2 n\right) .
\end{aligned}
$$

Note

$$
h_{n}\left(x_{2 k}\right)=\frac{1}{2 n} \int_{0}^{1} \ldots \int_{0}^{1} d x_{1} \ldots d x_{2 k-1} b\left(x_{1}\right) \ldots b\left(x_{2 k-1}\right) g_{2 k}\left(x_{1}, \ldots, x_{2 k} ; 2 n\right)
$$

then

$$
\alpha_{n}(k)=\int_{0}^{1} d x_{2 k} h_{n}\left(x_{2 k}\right) b\left(x_{2 k}\right) .
$$

Using Lemma 2:

$$
\begin{aligned}
g_{2 k}\left(x_{1}, \ldots, x_{2 k} ; 2 n\right) & =f_{2 k}\left(0, x_{1}-x_{2 k}, \ldots, x_{2 k-1}-x_{2 k} ; 2 n\right) \\
& =\prod_{l=1}^{2 k} \frac{\sin 2 n \pi\left(x_{l-1}-x_{l}\right)}{\sin \pi\left(x_{l-1}-x_{l}\right)}
\end{aligned}
$$

where we have $x_{0}=x_{2 k}$. 
Let $\pi\left(x_{l-1}-x_{l}\right)=z_{l}, l=1, \ldots, 2 k-1$ with $x_{0}=x_{2 k}$ then

$$
\begin{gathered}
x_{l}=x_{2 k}-\frac{1}{\pi}\left(z_{1}+\cdots+z_{l}\right), \quad l=1, \ldots, 2 k-1, \\
d x_{1} \ldots d x_{2 k-1}=\left(\frac{1}{\pi}\right)^{2 k-1} d z_{1} \ldots d z_{2 k-1}, \\
\frac{1}{2 n} g_{2 k}\left(x_{1}, \ldots, x_{2 k} ; 2 n\right)=\delta_{2 k-1}\left(z_{1}, \ldots, z_{2 k-1} ; 2 n\right) .
\end{gathered}
$$

Using Lemma 3 we have as $b(x)$ is a step function

i) $\lim _{n \rightarrow \infty} h_{n}\left(x_{2 k}\right) b\left(x_{2 k}\right)=b^{2 k}\left(x_{2 k}\right)$ almost everywhere furthermore

ii) $\left|b\left(x_{2 k}\right) h_{n}\left(x_{2 k}\right)\right| \leqq 1$.

i) and ii) assure the conditions of the Lebesgue dominated convergence theorem. Hence

$$
\alpha(k)=\lim _{n \rightarrow \infty} \alpha_{n}(k)=\int_{0}^{1} d x b^{2 k}(x) .
$$

In virtue of Weierstrasz's rule we can interchange lim and $\Sigma$ in [5]. Hence

$$
\begin{aligned}
s\left(\omega_{A}\right) & =\lim _{n \rightarrow \infty} s_{n}\left(\omega_{A}\right) \\
& =\log 2-\sum_{k=1}^{\infty} \frac{1}{2 k(2 k-1)} \int_{0}^{1} d x b^{2 k}(x) \\
& =-\int_{0}^{1} d x\left(\frac{1+b}{2}\right) \log \left(\frac{1+b}{2}\right)+\left(\frac{1-b}{2}\right) \log \left(\frac{1-b}{2}\right) \\
& =s^{\prime}(b) .
\end{aligned}
$$

$\hat{\mathscr{E}}$ is the set of multiplication operators with kernel $b(x)$ on $L^{2}\left(\mathbb{C}_{1}\right)$ such that $i b(x) f(x)=(\hat{A} f)(x), f \in L^{2}\left(\mathbb{C}_{1}\right)$ and such that $\omega_{A} \in \mathscr{E}$.

We define the functional $s^{\prime}: \hat{\mathscr{E}} \rightarrow[0, \log 2]$ by:

$$
s^{\prime}(b)=-\int_{0}^{1} d x\left(\frac{1+b}{2}\right) \log \left(\frac{1+b}{2}\right)+\left(\frac{1-b}{2}\right) \log \left(\frac{1-b}{2}\right) .
$$

Theorem 2. $s^{\prime}(b)=s\left(\omega_{A}\right) \quad \omega_{A} \in \mathscr{E}$ where $\hat{A}=i b$.

Proof. First the map $b \in \hat{\mathscr{E}} \rightarrow s^{\prime}(b)$ is continuous with respect to the norm topology (i.e. the supremum norm on the functions $b(x)$ ) (cfr. Lemma 1). Consider the complex $C^{*}$-algebra $\mathscr{F}$ generated by $\hat{\mathscr{E}}$, the 
subalgebra of step functions on $\mathbb{C}_{1}$ is dense in $\mathscr{F}$ because it separates the set of states on $\mathscr{F}$ (see [8], Théorème 11.3.1). Hence every function $b \in \hat{E}$ can be approximated in norm by a sequence $b_{n}$ of step functions of $\hat{E}$. Choose a sequence of step functions $b_{n} \in \hat{\mathscr{E}}$ converging in norm to $b \in \hat{\mathscr{E}}$ where $\hat{A}=i b$. Then the corresponding operators $A_{n} \in B(H)$, where $\hat{A}_{n}=i b_{n}$ converge also in norm to $A$ and:

By Lemma 4

$$
\left.s\left(\omega_{A}\right)=\lim _{n \rightarrow \infty} s\left(\omega_{A_{n}}\right) \quad \text { (Theorem } 1\right) .
$$

Hence

$$
s\left(\omega_{A_{n}}\right)=s^{\prime}\left(b_{n}\right) .
$$

$$
s\left(\omega_{A}\right)=\lim _{n \rightarrow \infty} s^{\prime}\left(b_{n}\right)=s^{\prime}(b) .
$$

Theorem 3. $\omega_{A} \in \mathscr{E} \rightarrow s\left(\omega_{A}\right)$ is not continuous with respect to the weak topology on $\mathscr{E}$.

Proof. Consider for instance the sequence of step functions $f_{n}$, $n=1,2, \ldots, f_{n} \in \hat{\mathscr{E}}$ defined by:

and

$$
\left.\begin{array}{rl}
f_{n+1}(x) & =f_{n}(2 x) \\
f_{n+1}(1-x) & =-f_{n+1}(x)
\end{array}\right\} \quad 0 \leqq x \leqq \frac{1}{2}
$$

$$
\begin{aligned}
f_{1}(x) & =1 & & 0<x<\frac{1}{2} \\
& =-1 & & \frac{1}{2}<x<1 \\
f_{1}(0) & =f_{1}\left(\frac{1}{2}\right)=0 . & &
\end{aligned}
$$

The multiplication operators

$$
\left(F_{n} \psi\right)(x)=i f_{n}(x) \psi(x) \quad \psi \in L^{2}\left(\mathbb{C}_{1}\right)
$$

converge weakly to zero. Therefore the corresponding quasi free states $\omega^{n}$ converge also weakly to the central state $\omega_{0} \in \mathscr{E}$, defined by:

But

$$
\omega_{0}(B(\psi) B(\phi))=s(\psi, \phi), \quad \psi, \phi \in H .
$$

$s\left(\omega^{n}\right)=s^{\prime}\left(f_{n}\right)=-\int_{0}^{1} d x\left(\frac{1+f_{n}}{2}\right) \log \left(\frac{1+f_{n}}{2}\right)+\left(\frac{1-f_{n}}{2}\right) \log \left(\frac{1-f_{n}}{2}\right)=0$.

Hence

$$
\lim _{n \rightarrow \infty} s\left(\omega^{n}\right)=0, \text { but } \quad s\left(\omega_{0}\right)=\log 2
$$

Acknowledgements. I wish to thank Prof. A. Verbeure for the great help he gave me during the preparation of this work. I thank also some other members of the institute with whom I had very helpfull discussions. 


\section{References}

1. Robinson, D. W., Ruelle, D.: Commun. math. Phys. 5, 288 (1967).

2. Lanford, O., Robinson, D. W.: J. Math. Phys. 9, 1120 (1968).

3. Shale, D., Stinespring, W. F.: Ann. Math. 80, 365 (1964).

4. Balslev, E., Manuceau, J., Verbeure, A.: Commun. math. Phys. 8, 315 (1968).

5. Hörmander, L. : Acta Math. 104, 93 (1960).

6. Verbeure, A.: Normal and locally normal quasi free states of Fermi systems. Cargèse Summer School Lecture Notes 1969. Ed.: D. Kastler. New York: Gordon and Breach.

7. Kato, T.: Perturbation theory for linear operators. Berlin-Heidelberg-New York: Springer 1966.

8. Dixmier,J.: Les $C^{*}$-algèbres et leurs représentations. Paris: Gauthier-Villars 1969.

M. Fannes

Instituut voor theoretische fysica

Dep. Nat. Celestijnenlaan 200D

B-3030 Heverlee, Belgium 